\title{
Tecnura
}

http://revistas.udistrital.edu.co/ojs/index.php/Tecnura/issue/view/640

DOI: http://dx.doi.org/10.14483/udistrital.jour.tecnura.2014.SE1.a02

INVESTIGACIÓN

\section{Cuantificación de existencias de compuestos bifenilos policlorados (PCB) en Colombia}

\section{Stocks quantification of polychlorinated biphenyl compounds (PCB) in Colombia}

\author{
Martha Lucía Vaca Bohórquez*, César Augusto García Ubaque**, \\ Juan Carlos García Ubaque***
}

Citation / Para citar este artículo: García Ubaque, C. A., García Ubaque, J. C., \& Vaca Bohórquez, M. L. (2014). Cuantificación de existencias de compuestos bifenilos policlorados (PCB) en Colombia. Revista Tecnura, Revista Tecnura, 39-44.

Fecha de recepción: 29 de noviembre de 2013 / Fecha de aceptación: 11 de julio de 2014

\begin{abstract}
Resumen
Los compuestos bifenilos policlorados, más conocidos como PCB, son sustancias de alta toxicidad tanto para el ser humano como para los ecosistemas. El Convenio de Estocolmo sobre Contaminantes Orgánicos Persistentes (COP), entre los que se encuentran los PCB, establece la necesidad de que los países tomen medidas para reducir o eliminar el uso de equipos eléctricos que aún funcionen con PCB. Por esta razón, el Ministerio de Ambiente de la República de Colombia (MINAMBIENTE) desarrolló un inventario sobre las existencias globales de PCB en uso. En este artículo se presentan la metodología seguida y los resultados consolidados de dicho inventario. El informe indica que las existencias estimadas de PCB en Colombia están entre 9.771 y 12.803 toneladas.
\end{abstract}

Palabras clave: bifenilos policlorados, compuestos orgánicos persistentes, ecotoxicidad, inventarios.

\begin{abstract}
Polychlorinated biphenyl compounds (PCB) are high toxicity substances for humans as well as for ecosystems. The Stockholm Convention on Persistent Organic Pollutants, including PCB, establishes the need for countries to reduce or eliminate the use of electrical equipment that still operates with PCB. Thus, the Ministry of Environment, Housing and Territorial Development in Colombia (MINAMBIENTE) conducted a preliminary inventory of PCB in use. This article presents the methodology and results of this inventory. The report shows that estimated stocks of PCB in Colombia are among 9.771 and 12.803 tons.
\end{abstract} Keywords: ecotoxicity, inventories, persistent organic compounds, polychlorinated biphenyls.

\footnotetext{
* Psicóloga, especialista en salud ocupacional, especialista en derecho ambiental, magíster en Business Administration, consultora independiente, Bogotá, Colombia.ml.vaca68@uniandes.edu.co

** Ingeniero civil, magíster en ingeniería ambiental, doctor en ingeniería; docente, Universidad Distrital Francisco José de Caldas, Bogotá, Colombia. cagarciau@udistrital.edu.co

*** Médico, magíster en salud pública, doctor en salud pública; docente, Universidad Nacional de Colombia, Bogotá, Colombia. jcgarciau@unal.edu.co
} 


\section{INTRODUCCIÓN}

Los bifenilos policlorados (PCB) son una clase de productos químicos orgánicos sintéticos, fabricados desde 1930, que debido a su estabilidad química son resistentes al fuego, tienen baja conductividad eléctrica, alta resistencia a la ruptura térmica, a oxidantes y otros productos químicos, por lo cual se han usado como fluidos dieléctricos, retardantes de llama, solventes, intercambiadores de calor, etc. (UNEP, 2003). Hacia 1970 se encontró que estas sustancias representaban una grave amenaza para la salud humana y el medio ambiente y poco a poco su producción se detuvo. Los efectos adversos asociados con la exposición de los PCB se dan en el sistema inmunológico, hígado, piel, sistema reproductor, aparato gastrointestinal y la glándula tiroides (Boix \& Cauli, 2012); (Chiu-Yueh, Tien-Shang, Kao-Chang, Pei-Chien, \& Yue Leon, 2011) (Remer \& Boekelheide, 2013).

Se estima que alrededor de 1,7 millones de toneladas de PCB se produjeron entre 1929 y 1989 y muchos de los equipos que contienen PCB están todavía en uso en algún lugar o almacenados a la espera de disposición final. Como los PCB una vez liberados al medio ambiente no se descomponen pero sí pueden viajar largas distancias, continúan planteando riesgos para la salud a los seres humanos, por lo cual es importante eliminar su uso y destruir los arsenales existentes. Las opciones de destrucción son principalmente la entrada de energía química o térmica a través de la ruptura de los enlaces moleculares y el método más común es la incineración de alta temperatura, aunque existen otros métodos no térmicos como la decloración (PNUMA, 2005) (Seeger, Timmis, \& Hofer, 1997) (Shimura, Koana, Fukuda, \& Kimbara, 1996) (Zanaroli, y otros, 2010) (Librando, y otros, 2013).

En respuesta a esta problemática, el Convenio de Estocolmo sobre Contaminantes Orgánicos Persistentes (COP) ha requerido a los países la adopción de medidas para reducir el uso y eliminar de una manera ambientalmente racional los equipos, los líquidos y los desechos contaminados con PCB tan pronto como sea posible, pero a más tardar en el año 2028 (MINAMBIENTE, 2007). Para esto, los países deberán comenzar a hacer esfuerzos decididos para identificar, etiquetar y retirar de uso los equipos con PCB y promover medidas para la reducción de la exposición y el riesgo para la salud humana y el ambiente. De hecho, la cuantificación e identificación de los PCB presentes en el medio ambiente que aún tienen contacto cotidiano con la población humana son objeto de numerosas investigaciones en los territorios de países fabricantes, como Estados Unidos, Italia, Alemania, China y Japón, entre otros (Delistraty, 2013) (Clarke, Porter, Marriot, \& Blackbeard, 2010) (Hopf, Ruder, \& Sucoop, 2009) (Bianco, y otros, 2009), así como en países solo consumidores (Mohammed, y otros, 2011) (Dodoo, Essumang, \& Johathan, 2013).

Este artículo presenta los resultados obtenidos en el Inventario Nacional de Existencias de Bifenilos Policlorados (PCB) en Colombia, realizado como parte del proyecto de asistencia técnica "Actividades Habilitadoras sobre Contaminantes Orgánicos Persistentes (COP) en Colombia" (MINAMBIENTE, 2007). El alcance del inventario comprendió la investigación a los sectores eléctricos del Sistema Interconectado Nacional (líneas mayores o iguales a $34,5 \mathrm{~kW}$, subestaciones, generadores y distribuidores asociados a estas líneas), empresas de gran consumo de energía (> $1 \mathrm{MW}$ ) de otros sectores y a los fabricantes, comercializadores y reparadores de equipos eléctricos, al año de referencia 2002.

\section{METODOLOGÍA}

La metodología seguida para este inventario siguió los lineamientos de la guía para la identificación de PCB y los materiales que contienen PCB de las Naciones Unidas (UNEP, 1999). Se siguieron estas etapas:

1. Recolección de información secundaria, mediante la consulta de otros inventarios efectuados en la región (Argentina, Bolivia, Brasil, Chile, México, Paraguay, Uruguay y Venezuela), registros de 
importaciones y exportaciones, empresas del sistema de interconexión eléctrica, autoridades ambientales locales y otras entidades gubernamentales.

2. Selección de una muestra estadística para la recolección de información directa. Se utilizó el muestreo aleatorio estratificado y por conglomerados. Se dividió la población en cinco estratos, de acuerdo con la capacidad y tipo de transformadores (ver la tabla 1).

Tabla 1. Clasificación por estratos.

\begin{tabular}{cl}
\hline Estrato & \multicolumn{1}{c}{ Descripción } \\
\hline 1 & Empresas generadoras netas. \\
\hline 2 & Empresas con $<1.000$ transformadores. \\
\hline 3 & Empresas entre 1.001 y 10.000 transformadores. \\
\hline 4 & Empresas entre 10.001 y 20.000 transformadores. \\
\hline 5 & Empresas con $>20.001$ transformadores. \\
\hline
\end{tabular}

Fuente: (MINAMBIENTE, 2007).

Para efectuar el cálculo del tamaño de la muestra se utilizó la ecuación (1).

$$
n=\frac{\sum_{h=1}^{1} W_{h}\left[\frac{N_{h} P_{h} Q_{h}}{N_{h}-1}\right]}{V(\hat{p})+\left(\left(1 / N\left\{\sum_{h=1}^{1} W_{h}\left[\frac{N_{h} P_{h} Q_{h}}{N_{h}-1}\right]\right\}\right)\right.}
$$

Donde:

1: Número de estratos

$\mathrm{N}_{h}$ : Universo o población de instalaciones en cada estrato

$\mathrm{N}$ : Universo o población total, corresponde a $N=\sum_{h=1} N_{h}$

$\mathrm{W}_{\mathrm{h}}$ : Corresponde a la relación $\frac{N_{h}}{N}$

P. Proporción de casos de interés en cada estrato, que se

$P_{h}:$ define como $p$ igual para todos los estratos en $10 \%$

$\mathrm{Q}_{h}:$ Se determina mediante la ecuación $Q_{h}=1-P_{h}$ Varianza esperada para el estimador del parámetro. Se obtiene con $v_{(f)}=\left(\frac{d}{Z_{\alpha / 2}}\right)^{2}$, donde $d=$ error de estima-

$V_{(\hat{p})}$ : ción de 0,01 $Z_{\alpha / 2}$ y $=$ percentil $(1-\alpha / 2) * 100$ de la distribución normal estándar. En este caso 1,64, que corresponde a un nivel de confianza de $90 \%$.
1. El territorio nacional se dividió en seis zonas: a) Costa Atlántica; b) Región Cundiboyacense y Meta; c) Eje Cafetero, Antioquia y Córdoba; d) Costa Pacífica y Valle del Cauca; e) Nariño, Cauca y Putumayo; f) Santanderes y Cesar.

2. Envío de cuestionarios para obtener información sobre las características de los equipos eléctricos existentes. Solamente los sectores eléctrico, manufactura e hidrocarburos reportaron tener existencias de PCB.

3. Realización de visitas de campo. Se visitaron algunas instalaciones con el fin de identificar las condiciones de almacenamiento de equipos eléctricos y aceites y determinar sitios potencialmente contaminados.

4. Cuantificación de existencias de PCB reportadas directamente. Se registró la información de residuos sólidos y aceites con concentraciones de PCB > 50 ppm o con PCB puro.

5. Realización de muestreos de PCB para validar información. Mediante estas mediciones se establecieron índices de probabilidad de contaminación de un transformador, de acuerdo con la marca, potencia, país y año de fabricación.

6. Realización del inventario estimado de PCB con base en proyecciones estadísticas de la información obtenida en el inventario directo y las mediciones efectuadas. Este paso se realizó en dos etapas: a) cálculo de los índices de contaminación de acuerdo con las características de cada equipo; b) proyección estadística de acuerdo con los índices calculados y el número y características de equipos reportados en cada región.

7. Estimación del total de equipos contaminados y el peso total de PCB.

8. Cuantificación del inventario consolidado de PCB en el país.

\section{RESULTADOS}

Los resultados obtenidos en el inventario se presentan en las tablas 2 a 5 . 
Tabla 2. Inventario directo de existencias de PCB en Colombia.

\begin{tabular}{|c|c|c|c|c|c|c|}
\hline \multirow[b]{2}{*}{ Sector } & \multicolumn{3}{|c|}{ Material sólido (kg) } & \multicolumn{2}{|c|}{ Aceite (kg) } & \multirow[b]{2}{*}{$\begin{array}{c}\text { Total } \\
(\mathbf{k g})\end{array}$} \\
\hline & $\begin{array}{c}\text { Carcasas de } \\
\text { transformadores }\end{array}$ & $\begin{array}{c}\text { Carcasas de } \\
\text { condensadores }\end{array}$ & $\begin{array}{l}\text { Residuos } \\
\text { sólidos }\end{array}$ & $\begin{array}{c}\text { Contenido de } \\
\text { transformadores y } \\
\text { condensadores }\end{array}$ & $\begin{array}{c}\text { Almacenado } \\
\text { en canecas }\end{array}$ & \\
\hline \multirow{2}{*}{ Eléctrico } & $388.154,7$ & 0 & 9.900 & $142.170,8$ & 28.624 & \multirow{2}{*}{$568.849,5$} \\
\hline & & Total & $398.054,7$ & Total & $170.794,8$ & \\
\hline \multirow{2}{*}{ Manufactura } & $195.109,7$ & 844 & 0 & $64.925,8$ & 1.926 & \multirow{2}{*}{$262.805,5$} \\
\hline & & Total & $195.953,7$ & Total & $66.851,8$ & \\
\hline \multirow{2}{*}{ Hidrocarburos } & $66.446,9$ & 0 & 0 & $28.553,1$ & 0 & \multirow{2}{*}{95.000} \\
\hline & & Total & $66.446,9$ & Total & $28.553,1$ & \\
\hline \multirow{2}{*}{ Total } & $649.711,3$ & 844 & 9.900 & $235.649,7$ & 30.550 & \multirow{2}{*}{926.655} \\
\hline & & Total & $660.455,3$ & Total & $266.199,7$ & \\
\hline
\end{tabular}

Fuente: (MINAMBIENTE, 2007).

Tabla 3. Resultados de muestreos de PCB.

\begin{tabular}{lccc}
\hline \multicolumn{1}{c}{ Tipo de equipo } & Número de muestras & $\begin{array}{c}\text { Resultado positivo } \\
\text { Kit Clor-N-Oil 50 }\end{array}$ & $\begin{array}{c}\text { Resultado negativo } \\
\text { Kit Clor-N-Oil 50 }\end{array}$ \\
\hline $\begin{array}{l}\text { Canecas con aceite dieléc- } \\
\text { trico }\end{array}$ & 27 & 0 & 27 \\
\hline Condensador & 1 & 1 & 0 \\
\hline Filtro termovacío & 1 & 0 & 1 \\
\hline Transformadores eléctricos & 1.494 & 150 & 1.344 \\
\hline
\end{tabular}

Fuente: (MINAMBIENTE, 2007).

Tabla 4. Estimación de existencias de PCB en los transformadores de todos los sectores, equipos en uso y en desuso.

\begin{tabular}{|c|c|c|c|c|c|c|c|c|c|}
\hline \multirow[t]{2}{*}{ Sector } & \multirow{2}{*}{$\begin{array}{l}\text { Transfor- } \\
\text { madores } \\
\text { en uso }\end{array}$} & \multirow{2}{*}{$\begin{array}{c}\text { Transfor- } \\
\text { madores } \\
\text { en desuso }\end{array}$} & \multirow[t]{2}{*}{$\begin{array}{c}\text { Total de } \\
\text { transformadores }\end{array}$} & \multicolumn{2}{|c|}{$\begin{array}{c}\text { Cantidad estimada } \\
\text { de transformadores } \\
\text { contaminados en uso }\end{array}$} & \multicolumn{2}{|c|}{$\begin{array}{c}\text { Cantidad estimada } \\
\text { de transformadores } \\
\text { contaminados en } \\
\text { desuso }\end{array}$} & \multicolumn{2}{|c|}{$\begin{array}{l}\text { Cantidad total } \\
\text { estimada de } \\
\text { transformadores } \\
\text { contaminados } \\
\end{array}$} \\
\hline & & & & $\begin{array}{c}\text { Escenario } 1 \\
\mathbf{0 , 0 5 8} \\
\end{array}$ & $\begin{array}{c}\text { Escenario } 2 \\
\mathbf{0 , 0 7 6} \\
\end{array}$ & $\begin{array}{c}\text { Escenario } 1 \\
\mathbf{0 , 0 5 8} \\
\end{array}$ & $\begin{array}{c}\text { Escenario } 2 \\
\mathbf{0 , 0 7 6} \\
\end{array}$ & $\begin{array}{c}\text { Escenario } 1 \\
\mathbf{0 , 0 5 8} \\
\end{array}$ & $\begin{array}{c}\text { Escenario } 2 \\
\mathbf{0 , 0 7 6} \\
\end{array}$ \\
\hline Transporte & 47 & 1 & 48 & 3 & 4 & 0 & 0 & 3 & 4 \\
\hline Minería & 3.713 & 115 & 3.828 & 215 & 282 & 7 & 9 & 222 & 291 \\
\hline Hidrocarburos & 957 & 30 & 987 & 56 & 73 & 2 & 2 & 57 & 75 \\
\hline Militar & 140 & 4 & 144 & 8 & 11 & 0 & 0 & 8 & 11 \\
\hline Eléctrico & 368.692 & 11.404 & 380.095 & 21.384 & 28.021 & 661 & 867 & 22.046 & 867 \\
\hline Total & 374.364 & 11.580 & 385.943 & 21.713 & 28.452 & 672 & 880 & 22.385 & 29.332 \\
\hline \multicolumn{4}{|c|}{ Peso promedio por transformador } & \multicolumn{6}{|c|}{$450 \mathrm{~kg}=0,45$ ton } \\
\hline \multicolumn{4}{|c|}{ Toneladas de equipos contaminados con PCB } & 9.771 & 12.803 & 302 & 396 & 10.073 & 13.199 \\
\hline
\end{tabular}

Fuente: (MINAMBIENTE, 2007). 
Tabla 5. Inventario preliminar de PCB existentes en Colombia.

\begin{tabular}{|c|c|c|}
\hline $\begin{array}{c}\text { Tipo de metodología } \\
\text { utilizada }\end{array}$ & Fuente de PCB & $\begin{array}{l}\text { Cantidad } \\
(\mathbf{t})\end{array}$ \\
\hline \multirow{4}{*}{ Estimación } & $\begin{array}{l}\text { Probable existencia de PCB puro en transformadores } \\
\text { en desuso }\end{array}$ & 683,27 \\
\hline & Probable existencia de PCB en transformadores en uso & 547,74 \\
\hline & $\begin{array}{l}\text { Probable existencia de PCB por contaminación en } \\
\text { transformadores en uso }\end{array}$ & entre 9.771 y 12.803 \\
\hline & $\begin{array}{l}\text { Probable existencia de PCB por contaminación en } \\
\text { transformadores en desuso }\end{array}$ & entre 302 y 396 \\
\hline $\begin{array}{l}\text { Recolección directa, reportes } \\
\text { y formularios }\end{array}$ & $\begin{array}{l}\text { Existencias de equipos, aceites y residuos contamina- } \\
\text { dos con PCB }\end{array}$ & 926,65 \\
\hline \multirow[b]{2}{*}{ Levantamiento de registros } & PCB importado al país en aceites lubricantes y equipos & 20,14 \\
\hline & $\begin{array}{l}\text { Condensadores importados al país antes del año } 1985, \\
\text { probablemente con PCB }\end{array}$ & $3.863,39$ \\
\hline
\end{tabular}

Fuente: (MINAMBIENTE, 2007).

De acuerdo con el cálculo de equipos evaluados sobre equipos que dieron positivo, se obtuvo un índice de contaminación por rango y potencia. Al analizar el total de los equipos considerados se obtuvo que el índice de contaminación nacional con PCB es 0,067 , que se sitúa en un rango entre 0,034 y 0,159 . Para un rango de confianza de $99 \%$, este índice debe situarse entre 0,058 y 0,076 .

\section{DISCUSIÓN}

De acuerdo con los resultados del cuadro $\mathrm{V}$, la existencia de PCB en Colombia está entre 9.771 y 12.803 toneladas. Otros países de la región como Venezuela, estiman sus existencias entre 6.500 y 10.000 toneladas; Brasil estima sus existencias en 80.000 toneladas y Uruguay en 4.150 toneladas (MINAMBIENTE, 2007).

Una de las observaciones que pueden destacarse es que el conocimiento acerca de la naturaleza de los PCB y sus probables efectos por causa de la exposición prolongada es aún muy limitado por parte de la población que los utiliza.

Debido a que no existe percepción del riesgo sobre los PCB, se encontró que son muy comunes las prácticas inadecuadas de manejo. En general, se observó en la población una gran despreocupación, lo que contribuye a favorecer la dispersión indiscriminada de los PCB en el ambiente, mediante prácticas como la reutilización de equipos, mangueras y tanques de almacenamiento.

Aunque algunas empresas del sector eléctrico ya han iniciado procesos de reconversión de sus transformadores, se hace necesario estructurar un plan de acción interinstitucional, suficientemente ambicioso para hacer un inventario más preciso y llevar a cabo la reconversión de equipos junto con la destrucción de los aceites con PCB recolectados, siguiendo las recomendaciones de la UNEP (UNEP, 2003). Este proceso debe ser monitorizado y evaluado periódicamente por parte de las entidades de control ambiental. De igual manera, sus resultados deben ser divulgados a la población en general.

\section{FINANCIAMIENTO}

Programa de las Naciones Unidas para el DesarroIlo, PNUD 


\section{REFERENCIAS}

Bianco, G.; Novario, G.; Bochicchio, D.; Anzilotta, G.; Palma, A., \& Cataldi, T. R. (2009). Polychlorinated biphenyls in contaminated soil samples evaluated by GC-ECD with dual column and GC-HRMS. Chemosphere, 104-112.

Boix, J., \& Cauli, O. (2012). Alterations of serotonin system by polyclorinated biphenyls exposure. Neurochemistry International, 809-816.

Chiu-Yueh, Y.; Tien-Shang, H.; Kao-Chang, L.; Pei-Chien, T., \& Yue Leon, G. (2011). Menstrual effects among women exposed to polychlorinated biphenyls and dibenzofurans. Environmental Research, 288-294.

Clarke, B. O.; Porter, N. A.; Marriot, P. J., \& Blackbeard, J. R. (2010). Investigating the levels and trends of organochlorine pesticides and polychlorinated biphenyl in sewage sludge. Environment International, 323-329.

Delistraty, D. (2013). Ecotoxicity and risk to human fish consumers of polychlorinated biphenyls in fish near the Hanfore Site (USA). Science of the Total Environment, 14-21.

Dodoo, D. K.; Essumang, D. K., \& Johathan, J. W. (2013). Accumulation profile and seasonal variations of polychlorinated biphenyls (PCBs) in bivalves Crassostrea tulipa (oysters) and Anadar senilis (mussels) at three different aquatic habitats in two seasons in Ghana. Ecotoxicology and Environmental Safety, 26-34.

Hopf, N. B.; Ruder, A. M., \& Sucoop, P. (2009). Background levels of polychlorinated biphenyls in the U.S. population. Science of the Total Environment, 6109-6119.

Librando, L.; Minniti, Z.; Acolla, M. L.; Cascioc, O.; Castelli, F., \& Sarpietro, M. G. (2013). Calorimetric evaluation of interaction and absorption of polychlorinated biphenyls by biomembrane models. Chemosphere, 791-796.

MINAMBIENTE (2007). Inventario preliminar de compuestos bifenilos policlorados en Colombia.
Bogotá: Ministerio de Ambiente, Vivienda y Desarrollo Territorial.

Mohammed, A.; Peterman, P.; Echols, K.; Feltz, K.; Tegerdine, G.; Manoo, A.; Orazio, C. (2011). Polychlorinated biphenyls (PCBs) and organochlorine pesticides (OCPs) in harbor sediments from Sea Lots, Port-of-Spain, Trinidad and Tobago. Marine Pollution Bulletin, 1324-1332.

PNUMA (2005). Eliminando los COP del mundo: guía del Convenio de Estocolmo sobre Contaminantes Orgánicos Persistentes. Ginebra: Programa de las Naciones Unidas para el Medio Ambiente.

Remer, M., \& Boekelheide, K. (2013). Multiple environmental chemical exposures to lead, mercury and polychlorinated biphenyls among childbearingaged women (NHANES 1999-2004): Body burden and risk factors. Environmental Research, 23-30.

Seeger, M.; Timmis, K. N., \& Hofer, B. (1997). Bacterial pathways for degradation of polychlorinated biphenyls. Marine Chemistry, 327-333.

Shimura, M.; Koana, T.; Fukuda, M., \& Kimbara, K. (1996). Complete degradation of polychlorinated biphenyls by a combination of ultraviolet and biological treatments. Journal of Fermentation and Bioengineering, 573-576.

UNEP (1999). Guidelines for the identification of PCBs and materials containing PCBs. Ginebra: United Nations Environment Programme.

UNEP (2003). Preparación de un plan nacional de manejo ambientalmente adecuado de los bifenilos policlorados $(P C B) y$ de equipos contaminados con PCB. Ginebra: United Nations Environment Programme.

Zanaroli, G.; Balloi, A.; Negroni, A.; Daffonchio, D.; Young, L. Y., \& Fava, F. (2010). Characterization of the microbial community from the marine sediment of the Venice lagoon capable of reductive dechlorination of coplanar polychlorinated biphenyls (PCBs). Journal of Hazardous Materials, 417-426.

\section{(C) $(1) \Theta$}

Tecnura • p-ISSN: 0123-921X • e-ISSN: 2248-7638• Edición especial $2014 \bullet$ pp. 39-44 